\title{
Integrability and Diffeomorphisms on Target Space
}

\author{
Christoph ADAM ${ }^{\dagger}$, Joaquin SANCHEZ-GUILLEN ${ }^{\dagger}$ and Andrzej WERESZCZYNSKI ${ }^{\ddagger}$ \\ $\dagger$ Department of Particle Physics, University of Santiago de Compostela, Spain \\ E-mail: adam@fpaxp1.usc.es, jsanchezguillen@gmail.com \\ ¥ Institute of Physics, Jagellonian University, Reymonta 4, 30-059 Krakow, Poland \\ E-mail: wereszczynski@th.if.uj.edu.pl
}

Received October 18, 2007, in final form December 10, 2007; Published online December 20, 2007

Original article is available at http://www.emis.de/journals/SIGMA/2007/123/

\begin{abstract}
We briefly review the concepts of generalized zero curvature conditions and integrability in higher dimensions, where integrability in this context is related to the existence of infinitely many conservation laws. Under certain assumptions, it turns out that these conservation laws are, in fact, generated by a class of geometric target space transformations, namely the volume-preserving diffeomorphisms. We classify the possible conservation laws of field theories for the case of a three-dimensional target space. Further, we discuss some explicit examples.
\end{abstract}

Key words: integrability; zero curvature; conservation laws; nonlinear field theories

2000 Mathematics Subject Classification: 37K05; 37K30; 37K40; 58E50; 81R12; 81T10

\section{Introduction}

Integrability, that is the existence of infinitely many conservation laws, has been an invaluable concept for the analysis of nonlinear field theories in $1+1$ dimensions. In higher dimensions, on the other hand, much less is known about nonlinear field theories. A general concept of integrability has not yet been developed there.

One proposal for integrability in higher dimensions was provided in [1], where the zero curvature representation of Shabat and Zakharov has been generalized to higher dimensions. Further, it was demonstrated in that paper that this proposal leads to nonlinear field theories which have either infinitely many conservation laws in the full theory, or which contain integrable subsectors, defined by some additional constraint equations on the fields, such that the solutions belonging to these subsectors have infinitely many conservation laws. This zero curvature representation, therefore, realizes the concept of integrability in higher-dimensional non-linear field theories in a specific and well-defined manner.

These methods have later been applied to specific models and to the analytic construction of both static and time-dependent solutions. For models with infinitely many conservation laws (the so-called AFZ model and related models), static and time-dependent solutions have been constructed, e.g., in [2, 3, 4, 5], and in [6, 7], respectively. Solutions in integrable subsectors of models which are, themselves, not integrable, have been constructed, e.g., in [8, 9, 10, 11] (the Nicole model and versions thereof) and in $[12,13]$ (diverse models on base space $S^{3}$ ). All these models share the property that their target space has dimension two.

A well-known nonlinear field theory with three-dimensional target space is the Skyrme model $[14,15]$ with target space $\mathrm{SU}(2)$ (or equivalently the three-sphere $S^{3}$ ). Further, this model

\footnotetext{
${ }^{\star}$ This paper is a contribution to the Proceedings of the Seventh International Conference "Symmetry in Nonlinear Mathematical Physics" (June 24-30, 2007, Kyiv, Ukraine). The full collection is available at http://www.emis.de/journals/SIGMA/symmetry2007.html
} 
contains an integrable subsector, and the simplest Skyrmion (i.e., the simplest soliton of the Skyrme model with baryon number equal to one) belongs to this integrable subsector, see [16].

In many cases, it turns out that most of the new conserved currents in models and their subsectors are Noether currents and generalizations thereof, i.e., they are related to transformations of the target space variables (see [17]). So a direct, geometric approach has been successfully undertaken to find those currents, first for models with two-dimensional target spaces [18, 19], and later also for three-dimensional target spaces [20].

It is the purpose of this review to give a short overview of some of these recent results on higher-dimensional integrability mentioned above, and to present some applications. Concretely, in Section 2 we briefly review the generalized curvature condition which was proposed in [1] as a possible way to generalize integrability to higher dimensions. In Section 3 we introduce volumepreserving diffeomorphisms on target space. For the case of three-dimensional target space we then classify for a wide class of Lagrangians all possible conservation laws, where the conserved currents in all cases are Noether currents of the volume-preserving target space diffeomorphisms. This section closely follows [20], but provides a slightly more refined classification, which turns out to be useful for applications. In Section 4 we study as an explicit example the Abelian projection of Yang-Mills dilaton theory, which turns out to be integrable, and where an infinite number of analytic static solutions exist. Here we closely follow the results of [21]. Section 5 contains a brief discussion. In the appendix we provide the calculational details of the classification of Section 3.

\section{Generalized zero curvature condition}

Here we briefly review the proposal for generalized integrability of [1], to which we refer for the details. The structure needed consists of a reducible Lie algebra $\tilde{\mathcal{G}}$ which is a direct sum of another Lie algebra $\mathcal{G}$ and an Abelian ideal $\mathcal{H}$,

$$
\tilde{\mathcal{G}}=\mathcal{G} \oplus \mathcal{H}
$$

together with a flat connection

$$
A_{\mu} \in \mathcal{G}, \quad \partial_{\mu} A_{\nu}-\partial_{\nu} A_{\mu}+\left[A_{\mu}, A_{\nu}\right]=0,
$$

and a covariantly constant vector field

$$
B_{\mu} \in \mathcal{H}, \quad \partial_{\mu} B^{\mu}+\left[A_{\mu}, B^{\mu}\right]=0
$$

with

$$
A_{\mu}=A_{\mu}^{a} T^{a}, \quad B_{\mu}=B_{\mu}^{\alpha} P^{\alpha}, \quad\left[P^{\alpha}, P^{\beta}\right]=0,
$$

where $T^{a}$ and $P^{\alpha}$ form a basis in $\mathcal{G}$ and $\mathcal{H}$, respectively. Further, $\mu=1, \ldots, d$ are base space indices.

To gain some intuition, let us first remark that equation (1) is just a generalization to higher dimensions of the zero curvature condition of Zakharov and Shabat in $1+1$ dimensions. Further, equation (2) in some sense just generalizes the Lax pair $\dot{L}=[L, M]$ to higher dimensions. Another important point is that equations (1), (2) are not chosen arbitrarily but may, in fact be derived from a generalized curvature condition as follows.

Firstly, the zero curvature condition equation (1) of Zakharov and Shabat may be derived as a consequence of the path independence of the Wilson line (or parallel transport) operator

$$
W=P \exp \left(\int_{0}^{\sigma} d \sigma^{\prime} A_{\mu} \frac{d x^{\mu}}{d \sigma^{\prime}}\right)
$$


where $P$ indicates the path ordering. In an analogous way, equations (1), (2) may be derived from the hypersurface independence of the following hypersurface ordered operator $V$ in $d$ dimensions,

$$
V=\tilde{P} \exp \left(\int_{\Sigma_{d-1}} d \sigma^{1} \cdots d \sigma^{d-1} W^{-1} \tilde{B}_{\mu_{1} \ldots \mu_{d-1}} W \frac{d x^{\mu_{1}}}{d \sigma^{1}} \cdots \frac{d x^{\mu_{d-1}}}{d \sigma^{d-1}}\right) .
$$

Here the $d-1$ form $\tilde{B}$ is the Hodge dual of the vector (one-form) of equation (2) (in fact, $\tilde{B}$ is the more natural object from the point of view of generalized integrability). Further, $\Sigma_{d-1}$ is a based, ordered, closed hypersurface with base point $x_{0} \equiv x\left(\sigma_{j}=0\right)$. $\tilde{P}$ is the hypersurface ordering, which we shall explain a bit more in a moment. So far this is a generalization of the non-Abelian Stokes calculus formulated in three dimensions by Schlesinger in 1927 [22].

The hypersurface independence of $V$, in turn, may be derived from the zero curvature condition for a connection $\mathcal{A}$ in higher loop space $\Omega^{n}\left(M, x_{0}\right)$ where

$$
\Omega^{n}\left(M, x_{0}\right)=\left\{\gamma: S^{n} \rightarrow M, \gamma(0, \ldots, 0)=x_{0}\right\}
$$

Explicitly, the connection $\mathcal{A}$ reads

$$
\mathcal{A}=\int_{\Sigma_{d-1}} d \sigma^{1} \cdots d \sigma^{d-2} W^{-1} \tilde{B}_{\mu_{1} \ldots \mu_{d-1}} W \frac{d x^{\mu_{1}}}{d \sigma^{1}} \cdots \frac{d x^{\mu_{d-2}}}{d \sigma^{d-2}} \delta x^{\mu_{d-1}},
$$

where $\delta x^{\mu_{d-1}}$ is the differential on higher loop space which provides an arbitrary infinitesimal variation of the higher loop. A closed ordered based hypersurface $\Sigma^{d-1}$ may be interpreted as a closed loop in loop space $\Omega^{d-2}$, and this observation allows to understand the hypersurface ordering. It is just ordinary path ordering of the corresponding ordinary loop in higher loop space $\Omega^{d-2}$.

We want to emphasize that the conditions equations (1), (2) are sufficient, local conditions for the zero curvature condition on the connection $\mathcal{A}$ of equation (3), but certainly they are not the most general ones.

After this brief review of the generalized zero curvature condition, we assume that equations (1), (2) hold and make the following additional simplifying assumptions that

- $\mathcal{G}$ is a semisimple Lie algebra (e.g. su(2)) with

$$
\left[T^{a}, T^{b}\right]=f_{c}^{a b} T^{c}
$$

and structure constants $f_{c}^{a b}$.

- $\mathcal{H}$ is a (in general, reducible) representation space of $\mathcal{G}$ :

$$
\left[T^{a}, P^{\alpha}\right]=R^{\alpha \beta}\left(T^{a}\right) P^{\beta} .
$$

- $A_{\mu}$ is explicitly flat:

$$
A_{\mu}=g^{-1} \partial_{\mu} g, \quad g \in G
$$

(where e.g. $G=\mathrm{SU}(2)$ ) such that only equation (2) provides a nontrivial condition $\left(D_{\mu} B^{\mu}=0\right)$.

- Under these conditions, the currents

$$
J_{\mu}=g B_{\mu} g^{-1}
$$

are automatically conserved, $\partial^{\mu} J_{\mu}=0$, and therefore the number of the conserved currents equals the dimension of the representation space $\mathcal{H}$, $\operatorname{dim} \mathcal{H}$. If $\operatorname{dim} \mathcal{H}=\infty$, then we say that the corresponding field theory is integrable. 
In general, the conserved currents of an integrable theory may be either Noether currents or may be related to hidden symmetries. Under the assumptions equations (4)-(7), however, the currents $J_{\mu}$ turn out to be Noether currents of geometric target space transformations, where the target space is spanned by the parameters of $g \in G$.

A first example for this structure is like follows.

- The Lie group $G$ is $\mathrm{SU}(2)$ where, however, its elements $g \in G$ are restricted to the equator of $\mathrm{SU}(2)$ such that the target space is two-dimensional.

- The representation space $\mathcal{H}$ is the space of representations of $\mathrm{SU}(2)$ with arbitrary integer angular momentum quantum number $l$, but magnetic quantum number $m$ restricted to \pm 1 ,

$$
\mathcal{H}=\left\{\text { reps. } R_{l m} \text { of } \mathrm{SU}(2), m= \pm 1, l=1, \ldots, \infty\right\} .
$$

- Then the conserved currents $J_{\mu}$ of equation (7) turn out to generate area preserving diffeomorphisms on the two-dimensional target space (which may be, e.g., the two-sphere $S^{2}$, but this depends on the Lagrangian).

A detailed discussion of this case may be found in [17], or in $[18,19]$.

Another class of theories with three-dimensional target spaces is obtained when the group element $g$ is assumed to take values in the full unrestricted group $\mathrm{SU}(2)$. There it turns out that the resulting conservation laws are generated by some subsets of the generators of volumepreserving diffeomorphisms on that target space. This case is discussed in the next section, where also a classification of the conservation laws of these theories is given. More details may be found in [20].

\section{Conservation laws for Skyrme-type models}

\subsection{Volume-preserving diffeomorphisms}

Let us start with a three-dimensional manifold (later to be identified with target space) with local coordinates $X^{i}$ and with a volume form which in local coordinates reads

$$
d V=h\left(X^{i}\right) d X^{1} \wedge d X^{2} \wedge d X^{3}
$$

where $h$ is the volume density. Further, a diffeomorphism is an infinitesimal transformation

$$
X^{i} \rightarrow X^{i}+\epsilon Y^{i}\left(X^{j}\right),
$$

where $\epsilon$ is infinitesimal, and the $Y^{i}$ are arbitrary functions of the $X^{j}$. A volume-preserving diffeomorphism has to obey

$$
\partial_{i}\left(h Y^{i}\right) \equiv \frac{\partial}{\partial X^{i}}\left(h Y^{i}\right)=0,
$$

in addition. The corresponding vector fields

$$
v^{(Y)}=Y^{i} \partial_{i}
$$

form a closed Lie algebra, that is

$$
\left[v^{(Y)}, v^{(\tilde{Y})}\right]=v^{(\tilde{\tilde{Y}})}
$$

such that

$$
\tilde{\tilde{Y}}^{i}=\left(\partial_{j} Y^{i}\right) \tilde{Y}^{j}-\left(\partial_{j} \tilde{Y}^{i}\right) Y^{j}, \quad \partial_{i}\left(h \tilde{\tilde{Y}}^{i}\right)=0
$$


is again a volume-preserving diffeomorphism. For later convenience we change coordinates according to

$$
\begin{aligned}
& u \equiv X^{1}+i X^{2}, \quad \xi \equiv X^{3}, \\
& Y^{u} \equiv Y^{1}+i Y^{2}, \quad Y^{\xi} \equiv Y^{3} .
\end{aligned}
$$

These new coordinates are especially useful for a parametrization of $g \in \mathrm{SU}(2)$,

$$
g=\exp (i \xi \vec{n} \cdot \vec{\sigma})=\cos \xi+i \sin \xi \vec{n} \cdot \vec{\sigma}
$$

provided that we also replace the unit vector field $\vec{n}$ by a complex field $u$ via stereographic projection

$$
\vec{n} \rightarrow u=\frac{n_{1}+i n_{2}}{1+n_{3}} .
$$

Further, we assume from now on the following form of the volume density $h=h(u \bar{u}, \xi)$ for simplicity. Finally, we interpret $u, \xi$ as target space variables of a Lagrangian field theory with general Lagrangian $\mathcal{L}\left(u, \bar{u}, \xi, u_{\mu}, \bar{u}_{\mu}, \xi_{\mu}\right)$, where $u_{\mu} \equiv \partial_{\mu} u$, etc., then the Noether currents corresponding to the vector fields $v^{(Y)}$ generating volume-preserving diffeomorphisms on target space are given by

$$
J_{\mu}^{(Y)}=Y^{u} \Pi_{\mu}+Y^{\bar{u}} \bar{\Pi}_{\mu}+Y^{\xi} P_{\mu}
$$

with the usual canonical four-momenta

$$
\Pi_{\mu} \equiv \partial_{u^{\mu}} \mathcal{L}, \quad P_{\mu} \equiv \partial_{\xi^{\mu}} \mathcal{L} .
$$

The charges $Q^{(Y)}=\int d^{3} \mathbf{r} J_{0}^{(Y)}$ generate a Lie algebra isomorphic to the algebra of the vector fields $v^{(Y)}$ via the Poisson bracket, as usual.

\subsection{Classification of conserved currents}

We now specialize to the class of Lagrangians

$$
\mathcal{L}(a, b, c, \xi, d, e),
$$

where

$$
a=u \bar{u}, \quad b=u^{\mu} \bar{u}_{\mu}, \quad d=\xi^{\mu} \xi_{\mu}, \quad c=\left(u^{\mu} \bar{u}_{\mu}\right)^{2}-u_{\mu}^{2} \bar{u}_{\nu}^{2}, \quad e=\xi^{\mu} u_{\mu} \xi^{\nu} \bar{u}_{\nu} .
$$

To motivate this choice let us mention that, e.g., the Skyrme model belongs to this class. Indeed, the Skyrme model has the Lagrangian $\mathcal{L}_{\mathrm{Sk}}=\frac{m^{2}}{2} \mathcal{L}_{2}-\lambda \mathcal{L}_{4}$ where

$$
\begin{aligned}
& \mathcal{L}_{2}=\operatorname{tr}\left(g^{-1} g_{\mu} g^{-1} g^{\mu}\right)=d+4 b \frac{\sin ^{2} \xi}{(1+a)^{2}}, \\
& \mathcal{L}_{4}=\operatorname{tr}\left[g^{-1} g_{\mu}, g^{-1} g_{\nu}\right]^{2}=\frac{\sin ^{2} \xi}{(1+a)^{2}}(b d-e)+\frac{\sin ^{4} \xi}{(1+a)^{4}} c
\end{aligned}
$$

and $g$ is the $\mathrm{SU}(2)$ group element of equation (8).

For the class of Lagrangians (11) we now want to find which subsets of the currents (10) are conserved under which conditions. The calculation is lengthy and is, therefore, relegated to the Appendix. Here, we present the resulting classification in the following four tables. In Table 1 the fields just obey the field equations and, therefore, there is a one to one correspondence between symmetries and conservation laws. In Tables $2-4$, on the other hand, the fields have to obey certain first order equations ("integrability conditions"), in addition. These integrability conditions are not of the Euler-Lagrange type and, therefore, there is no longer a one to one correspondence between symmetries and conservation laws, see [23] for a detailed discussion. 
Table 1.

\begin{tabular}{|l|l|}
\hline \multicolumn{1}{|c|}{ No integrability conditions } \\
\hline a) & no condition on $\mathcal{L}$. \\
& Generically there exists only one vector field $Y$ : \\
& $Y^{u}=i u, \quad Y^{\bar{u}}=-i \bar{u}, \quad Y^{\xi}=0$. \\
\hline b) & $\mathcal{L}=\mathcal{F}\left(h b, h^{2} c, d, h e\right)$. \\
& There exist finitely many $Y$ generating the \\
& isometries of the target space metric. \\
\hline c) & $\mathcal{L}_{b}=0$ and $\mathcal{L}_{e}=0$. \\
& $Y$ form the Abelian subalgebra $(\tilde{G}=\tilde{G}(a)):$ \\
& $Y^{u}=i u \tilde{G}_{a}, \quad Y^{\bar{u}}=-i \bar{u} \tilde{G}_{a}, \quad Y^{\xi}=0$. \\
\hline d) & conditions b) and c) on $\mathcal{L}$, \\
& and factorizing $h=h_{1}(a) h_{2}(\xi)$. \\
& $Y$ forms the non-Abelian $\operatorname{subalgebra}^{-1}($ for $G=G(u, \bar{u})):$ \\
& $Y^{u}=i h_{1}^{-1} G_{\bar{u}}, \quad Y^{\bar{u}}=-i h_{1}^{-1} G_{u}, \quad Y^{\xi}=0$. \\
\hline
\end{tabular}

Case a) corresponds to the symmetry $u \rightarrow e^{i \alpha} u$. Case b) implies that the Lagrangian can be expressed by the pullback of a certain target space metric, such that $h$ is the Riemannian volume density of that metric. One example for this case is the Skyrme model. One example for case d) is provided by the Abelian projection of Yang-Mills dilaton theory, which is discussed in Section 4.

Table 2.

\begin{tabular}{|ll|}
\hline & Integrability condition $u^{2} \bar{u}_{\mu}^{2}-\bar{u}^{2} u_{\mu}^{2}=0$ \\
\hline a) & $\mathcal{L}_{b}=0$ and $\mathcal{L}_{e}=0$. \\
& $Y$ forms the Abelian subalgebra $($ for $G=G(a, \xi)):$ \\
& $Y^{u}=i h^{-1} u G_{a}, \quad Y^{\bar{u}}=-i h^{-1} \bar{u} G_{a}, \quad Y^{\xi}=0$. \\
\hline b) & $\mathcal{L}_{e}=0$. \\
& $Y$ form the Abelian subalgebra $(\tilde{G}=\tilde{G}(a)):$ \\
& $Y^{u}=i u \tilde{G}_{a}, \quad Y^{\bar{u}}=-i \bar{u} \tilde{G}_{a}, \quad Y^{\xi}=0$. \\
\hline
\end{tabular}

The integrability condition $u^{2} \bar{u}_{\mu}^{2}-\bar{u}^{2} u_{\mu}^{2}=0$ may also be expressed like $\partial^{\mu}(\bmod (u)) \partial_{\mu}(\arg (u))=0$, which provides a more geometric interpretation.

\section{Example: Abelian projection of YM dilaton theory}

Here we want to demonstrate that the Abelian projection of Yang-Mills dilaton theory is integrable in our sense. It belongs, in fact, to case d) of Table 1. A more detailed discussion can be found in [21]. The Lagrangian of Yang-Mills dilaton theory is

$$
\mathcal{L}=\frac{1}{4}\left(2 \xi^{\mu} \xi_{\mu}-e^{-2 \kappa \xi} F^{a \mu \nu} F_{\mu \nu}^{a}\right),
$$

where $A_{\mu}^{a}$ is an $\mathrm{SU}(2)$ Yang-Mills field and $F_{\mu \nu}^{a}$ is the corresponding field strength. Next, we want to employ the Cho-Faddeev-Niemi decomposition of the gauge field,

$$
A_{\mu}^{a}=n^{a} C_{\mu}+\epsilon^{a b c} n_{\mu}^{b} n^{c}+W_{\mu}^{a},
$$

where $C_{\mu}$ is an Abelian gauge field, $n^{a}$ is a unit vector in color space, and the so-called "valence field" $W_{\mu}^{a}$ is perpendicular to $n^{a}$ in color space, $n^{a} W_{\mu}^{a}=0$. To be consistent, the decomposition fields have to obey the constraint

$$
\partial^{\mu} W_{\mu}^{a}+C_{\mu} \epsilon^{a b c} n^{b} W_{\mu}^{c}+n^{a} W_{\mu}^{b} n_{\mu}^{b}=0 .
$$


Table 3.

\begin{tabular}{|c|c|}
\hline & Integrability conditions $u^{\mu} \xi_{\mu}=0$ \\
\hline & $\begin{array}{l}\text { no condition on } \mathcal{L} \text {; or } \mathcal{L}=\mathcal{F}\left(h b, h^{2} c, d, h e\right) \text {. } \\
Y \text { forms the Abelian subalgebra }(\text { for } G=G(a, \xi)) \text { : } \\
Y^{u}=i h^{-1} u G_{a}, \quad Y^{\bar{u}}=-i h^{-1} \bar{u} G_{a}, \quad Y^{\xi}=0 . \\
\text { And the further integrability condition } u^{2} \bar{u}_{\mu}^{2}-\bar{u}^{2} u_{\mu}^{2} \text { holds. }\end{array}$ \\
\hline b) & $\begin{array}{l}\mathcal{L}_{b}=0 . \\
Y \text { forms the Abelian subalgebra }(\text { for } G=G(a, \xi)) \text { : } \\
Y^{u}=i h^{-1} u G_{a}, \quad Y^{\bar{u}}=-i h^{-1} \bar{u} G_{a}, \quad Y^{\xi}=0\end{array}$ \\
\hline & $\begin{array}{l}\mathcal{L}_{b}=0 \text { and } \mathcal{L}=\mathcal{F}\left(h b, h^{2} c, d, h e\right) . \\
Y \text { form the subset } Y_{\xi}^{\xi}=0 \text { (is not a subalgebra). }\end{array}$ \\
\hline & $\begin{array}{l}\mathcal{L}_{b}=0 \text { and } \mathcal{L}=\mathcal{F}\left(h b, h^{2} c, d, h e\right) \text { and } \mathcal{W}(\mathcal{L})=0 \text {. } \\
\text { no further condition on } Y .\end{array}$ \\
\hline
\end{tabular}

Case a) is obeyed by many configurations of the Skyrme model. E.g., the simplest Skyrmion with baryon number one as well as many ansätze for Skyrmion configurations satisfy the conditions of case a). Case d): the "weight number" $\mathcal{W}$ is defined for monomials of first derivatives of fields as $\mathcal{W}=\operatorname{power}\left(u_{\mu}\right)+$ $\operatorname{power}\left(\bar{u}_{\mu}\right)-2$ power $\left(\xi_{\mu}\right)$, which gives e.g. $\mathcal{W}(b)=2, \mathcal{W}(e)=-2$.

Table 4.

\begin{tabular}{|l|l|}
\hline & Integrability conditions $u_{\mu}^{2}=0$ and $u^{\mu} \xi_{\mu}=0$ \\
\hline a) & no condition on $\mathcal{L}$. \\
& $Y$ forms the Abelian subalgebra $($ for $G=G(a, \xi)):$ \\
& $Y^{u}=i h^{-1} u G_{a}, Y^{\bar{u}}=-i h^{-1} \bar{u} G_{a}, \quad Y^{\xi}=0$. \\
\hline b) $\quad \mathcal{L}=\mathcal{F}\left(h b, h^{2} c, d, h e\right)$ & \\
& $Y$ form the subset $Y_{\xi}^{\xi}=0($ is not a subalgebra). \\
\hline c) $\quad \mathcal{L}=\mathcal{F}\left(h b, h^{2} c, d, h e\right)$ and $\mathcal{W}(\mathcal{L})=0$. \\
& no further condition on $Y$.
\end{tabular}

Case b) is obeyed by many configurations of the Skyrme model. E.g., the simplest Skyrmion with baryon number one or the rational map ansätze for Skyrmion configurations satisfy the conditions of case b).

This constraint makes that the number of degrees of freedom of the original gauge field and of the decomposition match, and further, it provides the correct behaviour under gauge transformations for the decomposition fields, which infinitesimally read

$$
\delta n^{a}=\epsilon^{a b c} n^{b} \alpha^{c}, \quad \delta W_{\mu}^{a}=\epsilon^{a b c} W_{\mu}^{b} \alpha^{c}, \quad \delta C_{\mu}=n^{a} \alpha_{\mu}^{a} .
$$

In a next step, we perform the Abelian projection, which consists in setting the valence field equal to zero,

$$
W_{\mu}^{a}=0
$$

Observe that the Abelian projection is gauge invariant and obeys the constraint (12). The resulting gauge field $\hat{A}_{\mu}^{a}=n^{a} C_{\mu}+\epsilon^{a b c} n_{\mu}^{b} n^{c}$ still is a full $\mathrm{SU}(2)$ connection, but with Abelian field strength. The resulting Abelian projected Yang-Mills dilaton theory is already integrable, that is, it has infinitely many conserved currents, see [21] for details. Here we make the further simplifying assumption $C_{\mu} \equiv 0$ (which is no longer gauge invariant). The resulting Abelian 
projected Lagrangian is

$$
\mathcal{L}_{\mathrm{AP}}=\frac{1}{4}\left(2 \xi^{\mu} \xi_{\mu}-e^{-2 \kappa \xi} H^{\mu \nu} H_{\mu \nu}\right)
$$

with

$$
H_{\mu \nu}=\epsilon^{a b c} n^{a} n_{\mu}^{b} n_{\nu}^{c}
$$

or, after the stereographic projection (9),

$$
\mathcal{L}_{\mathrm{AP}}=\frac{1}{2} \xi^{\mu} \xi_{\mu}-2 e^{-2 \kappa \xi} \frac{\left(u^{\mu} \bar{u}_{\mu}\right)^{2}-u_{\mu}^{2} \bar{u}_{\nu}^{2}}{(1+u \bar{u})^{4}} \equiv \frac{1}{2} d-2 h^{2} c,
$$

where $h=h_{1}(a) h_{2}(\xi) \equiv(1+a)^{-2} e^{-\kappa \xi}$. It corresponds to case d) of Table 1 and has, therefore, infinitely many symmetries and infinitely many conservation laws.

We now want to use our explicitly integrable parametrization of the Abelian projection of Yang-Mills dilaton theory to discuss the problem of static solutions. For that purpose, we should first review the known results on that issue. It is known that there exist static, sphaleron type solutions in Yang-Mills dilaton theory. For the fully non-Abelian theory, solutions both for radially and cylindrically symmetric ansätze are known numerically, whereas for the Abelian subsector solutions for radially and cylindrically symmetric ansätze are known analytically. In the latter case, it is further known that the energy of the analytic solutions grows linearly with a certain integer $m$ from the ansatz (the magnetic quantum number). The latter fact points to the existence of a Bogomolny bound in the Abelian projection, but an ansatz-independent derivation of this Bogomolny bound has not yet been given in the literature.

In our integrable Abelian projection of YM dilaton theory, the analytic solutions may be calculated easily by quadratures, and the Bogomolny bound may be derived explicitly. Indeed, upon introducing spherical polar coordinates $(r, \theta, \varphi)$ in three-dimensional base space, the ansatz $\xi=\xi(r), u=v(\theta) \exp (i m \varphi)$ turns out to be consistent with the static field equations because of the base space symmetries of the theory. The resulting ordinary differential equations for $\xi(r)$ and $v(\theta)$ turn out to be solvable by quadratures, such that the corresponding exact analytic solutions may be calculated easily. The solvability by quadratures of the field equations might be related to the integrability of the theory. For details we refer to [21].

Finally, the Bogomolny bound may be derived easily within our parametrization. Indeed, we find for the energy corresponding to the Lagrangian (13) for static configurations

$$
\begin{aligned}
E_{\mathrm{AP}} & =\frac{1}{2} \int d^{3} \mathbf{r}\left((\nabla \xi)^{2}+e^{-2 \kappa \xi} \vec{H}^{2}\right)=\frac{1}{2} \int d^{3} \mathbf{r}\left(\nabla \xi-e^{-\kappa \xi} \vec{H}\right)^{2}+\int d^{3} \mathbf{r} e^{-\kappa \xi} \nabla \xi \cdot \vec{H} \\
& \geq \int d^{3} \mathbf{r} e^{-\kappa \xi} \nabla \xi \cdot \vec{H} \equiv E_{\mathrm{Bog} .}
\end{aligned}
$$

(where $\vec{H}$ is the Hodge dual of $H_{j k}$ ) and, therefore, the Bogomolny equation

$$
\nabla \xi-e^{-\kappa \xi} \vec{H}=0 .
$$

All the analytic static solutions mentioned above satisfy this equation and are, therefore, Bogomolny solutions. Further, the Bogomolny energy $E_{\text {Bog. }}$ may be expressed by the winding number of a map $\mathrm{S}^{3} \rightarrow \mathrm{S}^{3}$, see again [21].

To recapitulate, our main results for the Abelian projection of YM dilaton theory are that

- there exist infinitely many symmetries and infinitely many conserved currents,

- this fact may explain the infinitely many analytic solutions (this still is a conjecture, which exploits the analogy to the lower dimensional cases),

- there exist both a Bogomolny bound and a Bogomolny equation for static configurations, and the latter is solved by all known analytic solutions. 


\section{Discussion and outlook}

It was the purpose of this article to briefly review some recent developments in the attempts to generalize the concept of integrability to higher-dimensional nonlinear field theories. We gave a brief introduction to the general proposal for higher-dimensional integrability of [1] and then showed how, under certain additional assumptions, this higher-dimensional integrability is related to certain geometric target space transformations (concretely, volume preserving differomorphisms) which provide infinitely many conservation laws. We discussed in some more detail the case of a three-dimensional target space and, as a specific example, the Abelian projection of Yang-Mills dilaton theory and its static analytic solutions. Some more applications to specific theories have already been studied (see, e.g., the references quoted in the Introduction), which already demonstrates the usefulness and importance of the concept of generalized integrability for the study of higher-dimensional nonlinear field theories. There exist, however, many more applications which are still open to further investigation. One obvious application is the search for time-dependent solutions (e.g. $Q$-balls) in theories where till now only static solutions have been found (e.g. in the integrable submodel of Yang-Mills dilaton theory of the previous section). Another possibility for generalizations consists in the choice of larger groups $\mathcal{G}$ instead of $\mathrm{SU}(2)$ in the integrability construction discussed in Section 2. This leads to integrable theories with higher-dimensional target spaces. The search for an integrable submodel of Einstein Yang-Mills dilaton theory and for analytic solutions within this submodel would be an obvious candidate, especially as for this theory only numerical solutions are known so far.

The generalizations and further investigations mentioned here still deal with a connection which is trivially flat, $A_{\mu}=g^{-1} \partial_{\mu} g$, such that the zero curvature condition (1) of Section 2 is trivially fulfilled, and equation (2) remains the only nontrivial generalized zero curvature condition. A further possible generalization consists in treating equation (1) as a nontrivial condition, too, which generates nontrivial constraints on the connection. The resulting modified generalized integrability might then lead to nonlocal conserved currents and to conservation laws which are not generated by geometric transformations, as is well-known to be the case in $1+1$ dimensions. This line of investigation is, however, almost completely unexplored, and the above remarks are, therefore, mainly a proposal at the moment.

\section{A Appendix}

In this appendix we provide the details of the calculation of the conserved currents (10) for the class of Lagrangians (11). The Lagrangians $\mathcal{L}=\mathcal{L}(a, b, c, \xi, d, e)$ have the following canonical four-momenta

$$
\begin{aligned}
& \Pi_{\mu}=\mathcal{L}_{u^{\mu}}=\bar{u}_{\mu} \mathcal{L}_{b}+2\left(b \bar{u}_{\mu}-\bar{u}_{\nu}^{2} u_{\mu}\right) \mathcal{L}_{c}+\left(\xi^{\nu} \bar{u}_{\nu}\right) \xi_{\mu} \mathcal{L}_{e} \\
& P_{\mu}=\mathcal{L}_{\xi^{\mu}}=2 \xi_{\mu} \mathcal{L}_{d}+\left(\left(\xi^{\nu} \bar{u}_{\nu}\right) u_{\mu}+\left(\xi^{\nu} u_{\nu}\right) \bar{u}_{\mu}\right) \mathcal{L}_{e}
\end{aligned}
$$

field equations

$$
\partial^{\mu} \Pi_{\mu}=\mathcal{L}_{u}=\bar{u} \mathcal{L}_{a}, \quad \partial^{\mu} P_{\mu}=\mathcal{L}_{\xi},
$$

and we need the following useful identities

$$
\begin{aligned}
& u^{\mu} \Pi_{\mu}=b \mathcal{L}_{b}+2 c \mathcal{L}_{c}+e \mathcal{L}_{e} \\
& \bar{u}^{\mu} \Pi_{\mu}=\bar{u}_{\mu}^{2} \mathcal{L}_{b}+\left(\bar{u}_{\mu} \xi^{\mu}\right)^{2} \mathcal{L}_{e} \\
& \xi^{\mu} \Pi_{\mu}=\left(\xi^{\mu} \bar{u}_{\mu}\right) \mathcal{L}_{b}+2\left(b \xi^{\mu} \bar{u}_{\mu}-\bar{u}_{\nu}^{2} \xi^{\mu} u_{\mu}\right) \mathcal{L}_{c}+d \xi^{\mu} \bar{u}_{\mu} \mathcal{L}_{e} \\
& u^{\mu} P_{\mu}=2\left(\xi^{\mu} u_{\mu}\right) \mathcal{L}_{d}+\left(\left(\xi^{\mu} \bar{u}_{\mu}\right) u_{\nu}^{2}+b \xi^{\mu} u_{\mu}\right) \mathcal{L}_{e} \\
& \xi^{\mu} P_{\mu}=2 d \mathcal{L}_{d}+2 e \mathcal{L}_{e}
\end{aligned}
$$


Now we want to calculate the divergence of the Noether currents (10)

$$
\begin{aligned}
\partial^{\mu} J_{\mu}^{(Y)}= & \left(Y^{u}{ }_{u} u^{\mu}+Y^{u} \bar{u}^{\mu}+Y^{u}{ }_{\xi} \xi^{\mu}\right) \Pi_{\mu}+\left(Y_{u}^{\bar{u}} u^{\mu}+Y^{\bar{u}} \bar{u}^{\mu}+Y^{\bar{u}}{ }_{\xi} \xi^{\mu}\right) \bar{\Pi}_{\mu} \\
& +\left(Y^{\xi} u_{u} u^{\mu}+Y^{\xi} \bar{u}^{\mu}+Y^{\xi}{ }_{\xi} \xi^{\mu}\right) P_{\mu}+Y^{u} \partial^{\mu} \Pi_{\mu}+Y^{\bar{u}} \partial^{\mu} \bar{\Pi}_{\mu}+Y^{\xi} \partial^{\mu} P_{\mu} .
\end{aligned}
$$

After a lengthy but straight-forward calculation, and using

$$
Y^{u}{ }_{u}+Y^{\bar{u}}{ }_{\bar{u}}=h\left[\left(h^{-1}\right)_{u} Y^{u}+\left(h^{-1}\right)_{\bar{u}} Y^{\bar{u}}+\left(h^{-1}\right)_{\xi} Y^{\xi}\right]-Y_{\xi}^{\xi}
$$

(which easily follows from $\partial_{i}\left(h Y^{i}\right)=0$ ), we find

$$
\partial^{\mu} J_{\mu}^{(Y)}=\mathrm{I}+\mathrm{II}+\mathrm{III}
$$

where

$$
\begin{aligned}
\mathrm{I}= & h\left(\bar{u} Y^{u}+u Y^{\bar{u}}\right)\left[\left(h^{-1}\right)_{a} u^{\mu} \Pi_{\mu}+h^{-1} \mathcal{L}_{a}\right]+h Y^{\xi}\left[\left(h^{-1}\right)_{\xi} u^{\mu} \Pi_{\mu}+h^{-1} \mathcal{L}_{\xi}\right] \\
& +Y_{\xi}^{\xi}\left(\xi^{\mu} P_{\mu}-u^{\mu} \Pi_{\mu}\right),
\end{aligned}
$$

and

$$
\mathrm{II}=\left(Y_{\bar{u}}^{u} \bar{u}_{\mu}^{2}+Y^{\bar{u}}{ }_{u} u_{\mu}^{2}\right) \mathcal{L}_{b} .
$$

For the special choice

$$
Y^{u}=i h^{-1} u G_{a}, \quad Y^{\bar{u}}=-i h^{-1} \bar{u} G_{a}, \quad Y^{\xi}=0, \quad G=G(a, \xi)
$$

this simplifies to

$$
\mathrm{II}=\left[\partial_{a}\left(h^{-1} G_{a}\right)\right]\left(u^{2} \bar{u}_{\mu}^{2}-\bar{u}^{2} u_{\mu}^{2}\right) \mathcal{L}_{b} .
$$

Further,

$$
\begin{aligned}
\mathrm{III}= & \left(u^{\mu} \xi_{\mu}\right)^{2} \mathcal{L}_{e} Y^{\bar{u}}{ }_{u}-u_{\nu}^{2} \bar{u}^{\mu} \xi_{\mu}\left(2 \mathcal{L}_{c} Y^{\bar{u}}{ }_{\xi}-\mathcal{L}_{e} Y^{\xi}{ }_{u}\right) \\
& +u^{\mu} \xi_{\mu}\left[\left(\mathcal{L}_{b}+2 b \mathcal{L}_{c}+d \mathcal{L}_{e}\right) Y^{\bar{u}}{ }_{\xi}+\left(2 \mathcal{L}_{d}+b \mathcal{L}_{e}\right) Y^{\xi}{ }_{u}\right]+\text { h.c. }
\end{aligned}
$$

(Note that the above, correct, expression equation (16) differs from the corresponding one in [20] by one sign and one factor of two, which are incorrect in [20]. This small error in that reference has, however, absolutely no significance for the results of [20].) From these results it is not difficult to reconstruct the classification presented in Tables 1-4 of the main text. Let us focus on Table 1 for the moment. If no conditions are imposed on the Lagrangian, then the $Y^{i}$ have to obey $u Y^{\bar{u}}+\bar{u} Y^{u}=0$ and $Y^{\xi}=0$ together with $Y_{\xi}^{u}=0, Y^{u}{ }_{\bar{u}}=0$, etc, which has the only solution $Y^{u}=i u, Y^{\bar{u}}=-i \bar{u}$, which is just case a) of Table 1 . In case b), the condition $\mathcal{L}=\mathcal{F}\left(h b, h^{2} c, d, h e\right)$ implies that the first two terms in I, equation (15), are absent, but for general $\mathcal{F}\left(h b, h^{2} c, d, h e\right)$, it is still difficult to reconstruct the corresponding target space metric. However, if we restrict to the subclass of models $\mathcal{L}=\mathcal{F}\left[d+h b, 4 h(b d-e)+h^{2} c\right]$ (to which e.g. the Skyrme model belongs), then the target space metric is just

$$
d s^{2}=d \xi \otimes d \xi+h \frac{1}{2}(d u \otimes d \bar{u}+d \bar{u} \otimes d u)
$$

and it is not difficult to check that the resulting conditions on $Y^{i}$ in that case are just the Killing equations for the above target space metric. Further, cases c) and d) are easy to check, because in these cases most terms in the conservation equation (14) are zero. The same is true for the remaining tables, because most terms in (14) vanish because of the integrability conditions. For Tables 3 and 4 we add the remark that the condition that the "weight number" defined in Table 3 vanishes implies that the third term in equation (15) is zero. For additional details we refer to [20]. 


\section{Acknowledgement}

A.W. gratefully acknowledges support from Adam Krzyżanowski Fund and Jagiellonian University (grant WRBW 41/07). C.A. and J.S.-G. thank MCyT (Spain) and FEDER (FPA200501963), and support from Xunta de Galicia (grant PGIDIT06PXIB296182PR and Conselleria de Educacion). Further, C.A. acknowledges support from the Austrian START award project FWF-Y-137-TEC and from the FWF project P161 05 NO 5 of N.J. Mauser.

\section{References}

[1] Alvarez O., Ferreira L.A., Sanchez-Guillen J., A new approach to integrable theories in any dimension, Nuclear Phys. B 529 (1998), 689-736.

[2] Aratyn H., Ferreira L.A., Zimerman A., Toroidal solitons in (3+1)-dimensional integrable theories, Phys. Lett. B 456 (1999), 162-170.

[3] Aratyn H., Ferreira L.A., Zimerman A., Exact static soliton solutions of (3+1)-dimensional integrable theory with nonzero Hopf numbers, Phys. Rev. Lett. 83 (1999), 1723-1726.

[4] De Carli E., Ferreira L.A., A model for Hopfions on the space-time $S^{3} \times \mathbb{R}$, J. Math. Phys. 46 (2005), 012703, 10 pages, hep-th/0406244.

[5] Wereszczynski A., Integrability and Hopf solitons in models with explicitly broken $\mathrm{O}(3)$ symmetry, Eur. Phys. J. C Part. Fields 38 (2004), 261-265, hep-th/0405155.

[6] Ferreira L.A., Exact time dependent Hopf solitons in 3+1 dimensions, J. High Energy Phys. 2006 (2006), no. 3, 075, 9 pages, hep-th/0601235.

[7] Riserio do Bonfim A.C., Ferreira L.A., Spinning Hopf solitons on $S^{3} \times \mathbb{R}$, J. High Energy Phys. 2006 (2006), no. 3, 097, 12 pages, hep-th/0602234.

[8] Nicole D.A., Solitons with nonvanishing Hopf index, J. Phys. G 4 (1978), 1363-1369.

[9] Wereszczynski A., Toroidal solitons in Nicole-type models, Eur. Phys. J. C Part. Fields 41 (2005), 265-268, math-ph/0504008.

[10] Wereszczynski A., Generalized eikonal knots and new integrable dynamical systems, Phys. Lett. B 621 (2005), 201-207, hep-th/0508121.

[11] Adam C., Sanchez-Guillen J., Vazquez R.A., Wereszczynski A., Investigation of the Nicole model, J. Math. Phys. 47 (2006), 052302, 22 pages, hep-th/0602152.

[12] Ward R.S., Hopf solitons on $S^{3}$ and $\mathbf{R}^{3}$, Nonlinearity 12 (1999), 241-246.

[13] Adam C., Sanchez-Guillen J., Wereszczynski A., Hopf solitons and Hopf Q-balls on $S^{3}$, Eur. Phys. J. C Part. Fields 47 (2006), 513-524, hep-th/0602008.

[14] Skyrme T.H.R., A nonlinear field theory, Proc. R. Soc. Lond. A 260 (1961), 127-138.

[15] Skyrme T.H.R., A unified field theory of mesons and baryons, Nuclear Phys. 31 (1962), 556-569.

[16] Ferreira L.A., Sanchez-Guillen J., Infinite symmetries in the Skyrme model, Phys. Lett. B 504 (2001), 195-200, hep-th/0010168.

[17] Babelon O., Ferreira L.A., Integrability and conformal symmetry in higher dimensions: a model with exact Hopfion solutions, J. High Energy Phys. 2002 (2002), no. 11, 020, 26 pages.

[18] Adam C., Sanchez-Guillen J., Generalized integrability conditions and target space geometry, Phys. Lett. B 626 (2005), 235-242, hep-th/0508011.

[19] Adam C., Sanchez-Guillen J., Wereszczynski A., Integrability from an Abelian subgroup of the diffeomorphism group, J. Math. Phys. 47 (2006), 022303, 8 pages, hep-th/0511277.

[20] Adam C., Sanchez-Guillen J., Wereszczynski A., Conservation laws in Skyrme-type models, J. Math. Phys. 48 (2007), 032302, 16 pages, hep-th/0610227.

[21] Adam C., Sanchez-Guillen J., Wereszczynski A., Integrable subsystem of Yang-Mills dilaton theory, hep-th/0703224.

[22] Schlesinger L., Parallelverschiebung und Krümmungstensor, Math. Ann. 99 (1927), 413-434.

[23] Adam C., Sanchez-Guillen J., Symmetries of generalized soliton models and submodels on target space $S^{2}$, J. High Energy Phys. 2005 (2005), no. 1, 004, 15 pages, hep-th/0412028. 